\title{
Heidegger et la constitution onto-théologique de la métaphysique cartésienne
}

\author{
François Jaran \\ [Heidegger Studies, 2003/19, pp. 65-80]
}

Dans son ouvrage Sur le prisme métaphysique de Descartes ${ }^{1}$, Jean-Luc Marion s’interroge sur l'appartenance de la métaphysique cartésienne à l'onto-théologie ${ }^{2}$. En s'efforçant de rechercher une telle structure à la base de la prima philosophia cartésienne, Marion espère ainsi combler un vide dans l'œuvre de Heidegger : une confrontation avec la métaphysique de Descartes qui se tienne sous l'égide de l'onto-théologie.

Bien que Heidegger n'ait jamais tenté explicitement une herméneutique onto-théologique de cette pensée ${ }^{3}$, cela ne signifie point qu'il ait négligé les questions qu'une telle entreprise ne manquerait pas de soulever. Lorsque Heidegger aborde la pensée cartésienne (autour de Sein und Zeit tout d'abord, mais aussi dans de nombreux textes plus tardifs) et l'aborde comme métaphysique, peut-il éviter - si la métaphysique est essentiellement telle d'en interroger la structure onto-théo-logique? Les interprétations de la prima philosophia cartésienne qu'a livrées Heidegger des années vingt aux années quarante permettent de dresser un portrait de la position heideggérienne quant à une éventuelle « constitution ontothéologique » de cette métaphysique.

Le problème que soulève une telle entreprise est évident: qui, de l'ego ou de Dieu, doit être considéré comme le premier principe de la métaphysique cartésienne? Descartes annonce-t-il l'idéalisme de Fichte ou ne fait-il que répéter la metaphysica de Suárez? Cette bipolarité fondamentale de la pensée cartésienne est ce qui a mené Jean-Luc Marion à parler, dans le cas de Descartes, d'une «onto-théologie redoublée »" Or, cette même question semble aussi s'être présentée à Heidegger. Si à l'époque de Sein und Zeit (19201928), Heidegger affirme que l'ontologie cartésienne n'a aucunement dépassé celle de la

1. PUF, collection Épiméthée, Paris, 1986 - abrégé SPMD.

2. Longtemps avant la conférence Identität und Differenz. (1957), Heidegger employait déjà le schéma de l'onto-théologie. Voir, entre autres, Kant und das Problem der Metaphysik (1929: Gesamtausgabe - abrégée GA, Band 3, 220; tr. Kant et le problème de la métaphysique, 277), Hegels Phänomenologie des Geistes (1930/31: GA, 32, 140-146 et 183; tr. La "Phénoménologie de l'esprit» de Hegel, 156-162 et 196), Schelling: Vom Wesen der menschlichen Freibeit (1809) (1936: GA, 42, 88; tr. Schelling: Le traité de 1809 sur l'essence de la liberté bumaine, 96), Die seinsgeschichtliche Bestimmung des Nibilismus (1944-1946: publié in Nietzsche II, GA, 6.2, 313-315; tr. La détermination ontologico-historiale du nibilisme, publié in Nietzsche II, 279-280), Einleitung ₹u »Was ist Metaphysik?"« (1949 : publié in Wegmarken, GA, 9, 378-380; tr. Qu'est-ce que la métaphysique? Introduction, publié in Questions I et II, 39-41).

3. SPMD, 5 .

4. Ibid., $\int 10$. 
scolastique et sa compréhension de l'étant in quantum ens creatum ${ }^{1}$, les cours des années trente et quarante offrent une lecture de cette même pensée axée cette fois sur l'ego cogitans et sa compréhension de l'étant in quantum ens cogitatum.

Notre propos est ici de confronter les différentes interprétations de la pensée cartésienne qu'a proposées Heidegger ${ }^{2}$ aux figures onto-théologiques qu'emploie Jean-Luc Marion dans Sur le prisme métaphysique de Descartes ${ }^{3}$. Cet exercice nous permettra de montrer comment se modifie, au fil des années, la compréhension heideggérienne de la métaphysique de Descartes. Mais auparavant, il importe de rappeler la position que Marion développe dans son ouvrage Sur le prisme métaphysique de Descartes.

Cette thèse particulière - celle de «l'onto-théo-logie redoublée» - repose sur la présence, dans l'œuvre de Descartes, de deux paroles distinctes sur l'être de l'étant : cogitatio et causa. Ces deux manières de dire l'étant correspondent, selon l'auteur, à deux modes d'être qui s'établissent en deux ontologies distinctes, formant ainsi une métaphysique redoublée.

La première onto-théologie cartésienne est celle qui va naitre au fil des deux premières Méditations et que l'on peut placer sous le signe de la connaissance ou encore, de la cogitatio. C'est avec la découverte d'une vérité toute simple ("Je suis, j’existe», AT, IX, 19) et donc, de l'existence d'un ego cogitans qu'apparaît la première pensée métaphysique de Descartes : «L'existence de l'ego appuie (begründet) la manière d'être des cogitata; la manière d'être qui se manifeste dans les cogitata, en les révélant comme des étants, fonde (gründet) l'ego dans son existence privilégiée. Pareille fondation, double et croisée, satisfait à la lettre aux caractères de ce que Heidegger décelait sous les noms de "constitution onto-théologique de la métaphysique" " ${ }^{4}$. La manière d'être de l'étant in quantum ens cogitatum (onto-) et l'existence de l'ego au fondement de ce mode d'être (-théo-), comprises à chaque fois à

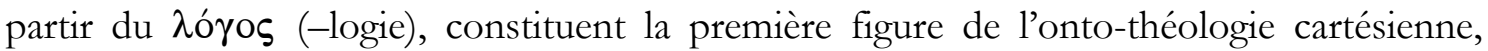
celle de la cogitatio.

Mais cette métaphysique est impuissante, selon Marion, à dire l'ensemble des thèmes traditionnels de la métaphysique. En effet, si l'ego a en lui l'idée de Dieu, il n'en possède pas pour autant la parfaite connaissance. L'idea Dei n'est qu'une cogitatio et ne peut, en sa finitude essentielle, recouvrir l'infini de la figure divine. La cogitatio, comme mode

1. D'où, rappelons-nous, l'incapacité de Descartes à penser correctement le mode d'être de l'ego.

2. Nous nous limitons à Sein und Zeit (1927), Die Frage nach dem Ding. Zu Kants Lebre von den transzendentalen Gründsätzen (1935/36) et Der europäische Nibilismus (1940). 10).

3. L'onto-théo-logie de la cogitatio, l'onto-théo-logie de la causa et l'onto-théo-logie redoublée (SPMD, \$ 8-

4. Ibid., 103. 
d'accès à l'étant, resterait donc fondamentalement en deçà d'une détermination correcte de l'essence de Dieu. L'insuffisance de l'ego cogitans est clairement exposée à la Méditation III où Descartes tente de démontrer l'existence de Dieu. À cette fin, il énonce que l'idée qu'il a d'une substance infinie ne peut émaner de son propre entendement, étant donné qu'il « doit y avoir pour le moins autant de réalité dans la cause efficiente et totale que dans son effet » (AT, IX, 28). Ce principe, «manifeste par la lumière naturelle », va donner aux Méditations une orientation fondamentalement nouvelle qui permet à une seconde onto-théologie celle de la causa - de se développer.

Ce «second visage» de la métaphysique cartésienne se déploie à partir de l'appréhension de l'étant in quantum ens creatum qui relègue au simple rang d'étant créé la res cogitans, principe de la première onto-théologie. Dépassant le domaine de la cognoscibilité de l'étant, cette seconde onto-théologie prétend traiter de l'existentia des étants. Alors que Descartes reconduit, au début de ses Méditations, l'étant à sa certitude épistémologique s'assurant ainsi d'un accès immédiat à l'objectivité de l'objet, il parvient, dans la suite de sa méditation, à sortir du cadre de la cogitatio et à atteindre la chose même, en tant qu'elle existe. Ouvrant l'accès à l'existence - «sens royal de l'être ${ }^{1}-$ de l'étant, cette seconde ontothéologie permet avant tout d'inclure Dieu et son existence à l'intérieur du champ de la métaphysique. Cette manière d'être de l'étant in quantum causatum sive creatum (onto-) et l'existence de Dieu au fondement de ce mode d'être (-théo-), comprises à chaque fois à

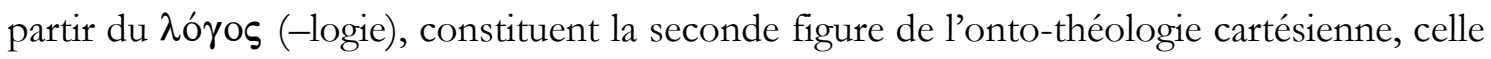
de la causa.

La solution que propose Marion - l'« onto-théo-logie redoublée » - au problème de la bipolarité de la métaphysique cartésienne, repose sur une décision quant au rapport qu'entretiennent les deux onto-théologies. Doit-on supposer qu'elles peuvent cohabiter sur un plan d'égalité dans la pensée cartésienne, dans un rapport de fondation réciproque? $\mathrm{Ne}$ doit-on pas plutôt suivre le cheminement même des Méditations - cheminement évidemment non aléatoire - et considérer l'onto-théologie de la cogitatio comme première, c'est-à-dire plus fondamentale? Ou alors, refusant de réduire l'infinité de Dieu à une pensée de l'ego, doit-on considérer que l'onto-théologie de la causa domine celle de la cogitatio? C'est cette dernière solution qu'a proposée Marion dans Sur le prisme métaphysique de Descartes. Afin de faire ressortir la position proprement heideggérienne, nous nous inspirerons de ces différents modèles de structures métaphysiques. Dans chacune de ses interprétations de la métaphysique cartésienne, Heidegger semble privilégier l'un des deux modèles onto- 
théologiques et regarder l'autre comme inessentiel. Seule la position qu'il défendra au milieu des années trente (WS 1935/36 : Die Frage nach dem Ding, analysé infra \2) permet de croire qu'il a envisagé la cohabitation que suppose l' « onto-théo-logie redoublée ».

\section{\1. L'onto-théologie de la causa: ens in quantum creatum}

Comme nous l'apprend le répertoire des cours professés par Heidegger ${ }^{1}$, les premières confrontations directes de Heidegger avec la pensée cartésienne remontent aussi loin qu'à l'été $1919^{2}$. Si ces séminaires ne sont pas publiés, il nous est cependant permis de gloser sur les motifs qui poussèrent Heidegger à aborder la pensée de Descartes. Il faut évidemment lier l'importance qu'accorde Heidegger à ce penseur à l'intérêt que lui portait son maittre Edmund Husserl qui, bien avant ses Méditations cartésiennes de 1929, avait déjà fait de Descartes un modèle à suivre pour sa phénoménologie ${ }^{3}$. Cette confrontation de Heidegger avec Descartes se déploie principalement à l'hiver 1923/24, alors que Heidegger donne son tout premier cours à Marbourg. Jusqu'à Sein und Zeit (1927), la critique de la pensée cartésienne s'articulera principalement autour de «l’indétermination ontologique » de l'ego.

Cette analyse - que les $\int 6$ et 10 de Sein und Zeit ont rendue classique - soutient que, s'appuyant sur l'évidence qui caractérise généralement l'idée d'« être », Descartes n’a pas cru bon de s'arrêter sur sa signification. Dans le cogito, ergo sum, Descartes ne s’intéresse qu’à la signification du penser et laisse fondamentalement indéterminée la manière d'être de l'ego, son sum $^{4}$. Privilégiant la certitude de l'ego, Descartes néglige d'en questionner la teneur en être. Mais bien que l'aveuglante clarté de cette évidence tienne Descartes éloigné du problème de la structure ontologique de l'ego, cela ne dispense pas sa pensée - dans la

1. Dressé par W. J. Richardson dans Heidegger. Through Phenomenology to Thought, Martinus Nijhoff, La Haye, 1974, 661-671, puis complété par T. Kisiel dans The Genesis of Heidegger's «Being \& Time », University of California Press, Berkeley, 1993, 461-468.

2. Heidegger a donné, au semestre d'été 1919, un séminaire intitulé Einfübrung in die Phänomenologie im Anscbluß an Descartes' Meditationes (inédit). Entre 1919 et 1930, Heidegger a consacré quatre séminaires (SS 1919, WS 1920/21, SS 1925 et WS 1929/30, tous inédits) et un cours (WS 1923/24: Einführung in die phänomenologische Forschung, $G A, 17)$ à la pensée cartésienne. Contrairement à ce que l'on a l'habitude de croire, Heidegger a donc beaucoup étudié - du moins, dans ces années-là - la philosophie cartésienne.

3. Voir à ce sujet J.-L. Marion, «L'ego et le Dasein» in Réduction et donation, PUF, Collection Épiméthée, Paris, 1989.

4. «Descartes, auquel on attribue la découverte du cogito sum comme base de départ du questionnement philosophique des temps modernes, fit porter sa recherche sur le cogitare de l'ego - dans certaines limites. En revanche il laisse le sum complètement en dehors du débat (völlig unerörtert) bien que le sum ait pour lui une position de départ tout aussi originale que le cogito » (Sein und Zeit-abrégé $S$. u. Z., 45-46; les références pour cet ouvrage sont données suivant le texte de la dixième édition, Max Niemeyer, Tübingen, 1963; tr. Être et temps, 78). 
mesure où elle est métaphysique, c'est à dire onto-théologique - de véhiculer une compréhension déterminée de l'être de l'étant. Or, le seul mode d'être que connaît Descartes est celui de l'objet rencontré dans le monde, celui de la chose (res). Ayant trouvé un fondement à sa pensée en l'ego cogitans - ontologiquement insuffisant, selon Heidegger -, Descartes croit pouvoir se soustraire aux préoccupations métaphysiques traditionnelles concernant la question de l'étant. Mais de cette façon, Descartes ne ferait qu'assujettir sa « théorie de la connaissance » à une compréhension de l'être qu'il n'a ni choisie ni tenté de justifier, faute de s'en être soucié.

Ce mode d'être des choses - au nombre desquelles on compte la res cogitans - que Descartes manque de questionner est puisé à même la tradition dans le concept de substantialité: "L'être d'un étant ne tenant qu'à lui-même répond au nom de substantia. L'expression veut tantôt dire l'être d'un étant en tant que substance, la substantialité, tantôt l'étant lui-même, une substance $»^{1}$. Or, Descartes définit la substance (c'est-à-dire l'étant luimême) ainsi : «Lorsque nous concevons la substance, nous concevons seulement une chose qui existe en telle façon qu'elle n'a besoin que de soi-même pour exister » (Principes de la philosophie, I, $\left.\sqrt{\int} 51, A T, \mathrm{IX}, \mathrm{II}, 47\right)^{2}$. Cet accent mis sur l'absence de besoin de la substance (son autarcie : « nulla alia re indigeat ad existendum ») manifeste l'horizon au sein duquel est compris l'être : l'étant par excellence (le $\theta \varepsilon \hat{\imath} 0 v$ ) se caractérise par une parfaite absence de besoin (ens perfectissimum, Dieu) et l'étant en tant que tel (l'ồ ר̣̂ ôv), qui «a besoin d'être produit au sens le plus large du terme, et d'être conservé »" ${ }^{3}$, est appréhendé in quantum ens creatum $^{4}$.

La compréhension cartésienne de l'être se réduit donc, selon Sein und Zeit, à ce que la scolastique avait développé avant lui ${ }^{5}$. Considérant alors la philosophie cartésienne selon

1. Ibid., 89-90; tr. 128.

2. «Per substantiam nihil aliud intelligere possumus, quam rem quae ita existit, ut nulla alia re indigeat ad existendum ». La traduction allemande de Heidegger reste cependant plus proche du texte latin que celle de l'édition française : «Unter Substanz können wir nichts anderes verstehen als ein Seiendes, das so ist, daß es, um ₹и sein, keines anderen Seienden bedarf » (S. u. Z., 92).

3. Ibid..

4. Notons qu'il ne s'agit pas de la seule interprétation possible de la doctrine cartésienne de la substance (celle des Principes de la philosophie, I, \51-54). Marion, dans Sur la théologie blanche de Descartes (PUF, Paris, 1981), 111 sq., offre une lecture plus juste de ce texte.

5. Dans Sein und Zeit, Heidegger écrit en fait que "pour ce qui est du travail en profondeur sur ce problème ontologique [celui de l'être], Descartes reste loin en deçà de la Scolastique, il esquive même la question» (93; tr. 132). Dans le cours du semestre d'été 1925, Prolegomena zur Geschichte des Zeitbegriffs, 
son aspect scolastique et son silence quant à la structure ontologique de l'ego cogitans, Heidegger soutient que la métaphysique cartésienne s'articule autour du concept de substantialité, mode d'être fondamentalement « hétérogène » à l'ego. Or, ce n'est qu'à partir de ce point de vue qu'est possible la critique concernant l'indétermination ontologique de l'ego. En effet, l'insuffisance ontologique de l'ego est due à l'absence d'un questionnement portant sur sa teneur en être. C'est cette carence du sujet moderne (effectif de Descartes à Husserl) que Heidegger espère corriger avec l'Analytique du Dasein. Mais cette caractérisation de l'ego comme substantia finita ou comme ens creatum est-elle aussi essentielle que le soutient alors Heidegger? Descartes n'a-t-il pas bouleversé l'histoire de la philosophie en faisant de l'ego cogitans le premier principe d'une nouvelle métaphysique?

\section{\2. Une onto-théologie redoublée?}

Ce portrait de la pensée cartésienne apparait, comme nous venons de le voir, dans le cadre d'une critique concernant l'incapacité de Descartes à repenser la métaphysique après la découverte de l'ego cogitans. Descartes possède alors tous les outils pour déployer une nouvelle ontologie mais se borne à une récupération des concepts scolastiques, manquant par là d'élucider le sum de l'ego. Car, avec ce renversement du questionnement philosophique de la nature à l'ego, « on s'attendrait à ce que dorénavant l'ontologie prenne pour étant exemplaire le sujet et qu'elle interprète le concept d'être eu égard au mode d'être qui appartient au sujet; on s'attendrait à ce que désormais le mode d'être du sujet devienne un problème ontologique $»^{1}$. Heidegger soutient donc, à la fin des années vingt ${ }^{2}$, que la pensée cartésienne ne représente aucunement un point tournant dans l'histoire de la pensée de l'être.

Mais Heidegger ne va pas en rester à cette lecture critique du texte cartésien. Dès le semestre d'hiver $1935 / 36^{3}$, il exposera une nouvelle interprétation du virage moderne davantage axée sur la nouveauté des Temps Modernes que sur la reprise de l'ontologie traditionnelle. Consacré à la Critique de la raison pure, ce cours développe l’idée - déjà

Heidegger était moins sévère à l'endroit de Descartes en affirmant que celui-ci, plutôt que d'esquiver la question, se rangeait, bien que cela ne soit pas dit spécifiquement, du côté de l'École. Voir GA, 20, 234; tr. History of the Concept of Time, 174.

1. Die Grundprobleme der Phänomenologie, GA, 24, 174; tr. Les problèmes fondamentaux de la phénoménologie, 155. Nous soulignons.

2. Voir GA, 24 (§13) et Die Grundbegriffe der Metaphysik. Welt - Endlichkeit - Einsamkeit, GA, 29/30 (\$ 14); tr. Les problèmes fondamentaux de la métaphysique. Monde - finitude - solitude.

3. Die Frage nach dem Ding. Zu Kants Lehre von den transzendentalen Grundsätzen, GA, 41; tr. Qu'est-ce qu'une chose? 
esquissée au $\int 69$ de Sein und Zeit - selon laquelle la métaphysique moderne est en son fondement guidée par le projet mathématique de la nature. Cette thèse va pousser Heidegger à reconsidérer l'avènement de la métaphysique moderne en tenant davantage compte du rôle qu'y joue l'orientation sur le sujet.

Tentant de caractériser la genèse ontologique de la physique mathématique et le projet qui ouvre l'a priori nécessaire à sa formation, Heidegger écrivait dans Sein und Zeit : « [Le projet mathématique] dévoile d'entrée de jeu un étant constamment là-devant (ein ständig Vorbandenes) (la matière) et ouvre l'horizon où le regard va se diriger principalement sur ses moments constitutifs déterminables quantitativement (mouvement, force, lieu et temps) ${ }^{1}$. Ce projet consiste donc en une nouvelle approche de l'étant qui porte avec elle une nouvelle compréhension de son être, une nouvelle possibilité de vérité (certitude), un nouveau type de fondation et de preuve, une nouvelle méthode ${ }^{2}$, etc. Le caractère mathématique de la science moderne n'est donc pas une conséquence de sa mise en forme par Descartes mais constitue, en réalité, le fondement préalable à la pensée cartésienne. Conformément au libre projet du monde qui caractérise la Renaissance, Descartes cherche et trouve une certitude première et inébranlable qui puisse répondre aux attentes du "géomètre ${ }^{3}$. C'est dans les écrits de Descartes que trouve sa fondation métaphysique l'acceptation de la vérité de l'étant que porte le projet mathématique - à savoir la certitude. C’est dire qu'avec Descartes, le questionnement métaphysique prend une autre tournure et qu'une nouvelle réponse à la question de l'étant est fournie.

Cette réponse, Descartes la déploie dans ses Meditationes de prima philosophia. Traduit en français par «Les Méditations métaphysiques», le titre original latin témoigne de la volonté de Descartes de s'inscrire ouvertement à l'intérieur de cette longue tradition philosophique

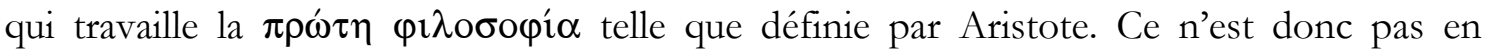
premier lieu une théorie de la connaissance que Descartes souhaite fonder, mais une nouvelle philosophie première qui réponde à la prétention mathématique et à ses exigences. Avec Descartes, le mathématique réussit pour la première fois à pénétrer l'intérieur de la réflexion ontologique.

1. S. u. Z., $362 ;$ tr. 425.

2. Ibid., 362-363; tr. 425.

3. Les échos de cette nouvelle liberté qu'exige le projet mathématique se font entendre jusque dans la philosophie pratique de Kant sous les idées de «loi morale» et d'«autonomie de la volonté », et dans le concept hobbesien de «contrat social». Nous la retrouvons aussi dans le mente concipere («se représenter en esprit») de Galilée (Discorsi, 1638) qui décide à l'avance, «en esprit », de ce qui vaut comme la choséité de la chose et s'y tient par la suite dans son appréhension de l'étant (voir $G A, 41,91-92 ;$ tr. 102). Cette liberté nouvelle, caractéristique de l'esprit de la Renaissance, se définit par un enchaînement volontaire et complet aux principes que l'on s'impose soi-même. 
Or, la philosophie cartésienne - à l'instar de l'ensemble de la tradition métaphysique - n'aborde jamais la question de l'être de l'étant de façon immédiate. Un long détour par une problématique méthodologique et un questionnement sur l'essence de la vérité servent de préambule à cette philosophie première. C'est tout d'abord dans les Regulae ad directionem ingenii que se manifeste de façon évidente le trait mathématique de la nouvelle pensée ${ }^{1}$ : «Dans la dénomination "règles" (Regulae) aussi bien que dans la prise en considération de la libre détermination interne de l'esprit (ad directionem ingenii), s'exprime déjà, dans le seul titre, le trait mathématique-métaphysique fondamental $»^{2}$. Cet ensemble de règles, découvert de façon a priori dans l'entendement, fonde une mathesis universalis, une science unique et normative qui puisse rendre compte de la totalité de l'étant et de sa connaissance. Or, c'est l'hypothèse de l'existence d'un tel ensemble de règles - aucunement évident de prime abord - qui témoigne du caractère vraiment mathématique de cette prima philosophia.

Par une analyse ${ }^{3}$ des Regulae $\mathrm{III}^{\mathrm{e}}$, IV et $\mathrm{V}^{\mathrm{e}}$, Heidegger caractérise cette mathesis universalis, cette « méthode en vue de la vérité des choses » (Reg. IV) comme devant s'appuyer sur un fond certain et progresser par déduction. Elle a donc besoin d'axiomes insignes qui puissent à eux seuls assurer la solidité du sol sur lequel elle se tient. Or, selon l'ensemble de la tradition philosophique, c'est la proposition qui est le lieu de la vérité de l'étant. C'est donc comme proposition que devra se présenter l'axiome de la nouvelle philosophie. Cependant, il est de la nature de la proposition d'énoncer quelque chose sur l'essence d'une chose, de contenir et de retenir (enthalten und behalten) ce que les choses sont ${ }^{4}$. Or, cette proposition de fond (Grundsatz) dont la prétention est de fonder notre connaissance de l'étant ne peut être précédée d'aucune chose. Le contenu de cette proposition insigne ne peut être reçu de l'extérieur mais doit «se livrer d'abord lui-même en tant que tel dans cette proposition originelle (ursprünglichen Satz), et y être posé » ${ }^{5}$.

La seule chose qui résiste à cette nouvelle fondation du savoir est l'énoncer, le poser, la proposition. Mais cet énoncer sera vide puisqu'il ne peut se référer à rien si ce n'est qu'à lui-même. L'axiome recherché doit mettre en évidence ce que celui qui énonce

1. Heidegger écrit alors que «seul celui qui, réellement et longuement, a médité de part en part jusque dans ses recoins les plus glacés cet écrit sobre et sans égard (rücksichtslos nüchterne Schrift), se met en état d'acquérir le pressentiment de ce qui est en marche dans la science moderne » (GA, 41, 102; tr. 111). Mais la petite quantité d'études qu'a consacrées Heidegger à cet écrit ne rend pas justice à l'importance qu'il lui accorde ici. Outre l'analyse des règles III, IV et V présentée dans ce cours, Heidegger ne traite de ce texte qu'à l'hiver 1923/24 (GA, 17) où il traite des règles II, III, IV, VI et XII et dans le cours Le nibilisme européen (GA, 6.2) de 1940 où le titre de la règle IV vient appuyer la description de la pensée de Descartes.

2. $G A, 41,101 ;$ tr. 111.

3. Ibid., 101-104; tr. 111-113.

4. Ibid., 103; tr. 113.

5. Ibid., 104; tr. 113. 
possède déjà ${ }^{1}$, afin d'être une pensée absolument mathématique. Le fundamentum de la connaissance est donc découvert par un retour de la pensée sur elle-même qui prend ainsi connaissance du penser lui-même et de son essence : «Tout énoncer, tout penser, quel que puisse être ce sur quoi il énonce, et en quelque sens que ce soit, est à chaque fois un "Je pense" $»^{2}$.

Heidegger a donc relativisé la position qu'il défendait dans Sein und Zeit ${ }^{3}$ selon laquelle Descartes n'aurait pas su penser à nouveaux frais la métaphysique et sa fondation. Bien que Descartes ne soit pas à l'origine du projet mathématique, ce sont ses écrits qui permettent à ce projet de trouver une légitimation au niveau métaphysique. L'être de l'étant ayant dorénavant pour fondement le «Je pense », une nouvelle métaphysique - celle de la subjectivité - peut désormais se déployer.

Mais même si l'ego cogitans acquiert par là un rôle beaucoup plus important, nous devons noter que la représentation chrétienne de l'étant (in quantum ens creatum) n'est pas moins conservée ${ }^{4}$. La métaphysique moderne, une métaphysique chrétienne formée à partir des principes et exigences de la raison pure, répond donc, soutient alors Heidegger, à une double structure : la représentation de l'étant en tant qu'ens creatum et le trait mathématique fondé en l'ego cogitans. "Dans la mesure où Dieu comme créateur est la cause et le fondement de tout étant, le comment, la manière d'interroger est orientée au préalable sur ce principe $»^{5}$. Mais il ne s'agit pas du seul principe : «Inversement le mathématique n'est pas seulement une forme plaquée sur le contenu chrétien, il relève lui-même du contenu ». Le principe du Je devient ainsi «principe directeur» désignant ainsi «le domaine auquel renvoient toutes les propositions métaphysiques et dont elles proviennent » ${ }^{6}$. Peut-on voir ici se profiler une tentative de réconcilier les deux figures de l'onto-théologie telles que les a identifiées Marion? Heidegger avait-il déjà envisagé « l'onto-théo-logie redoublée »?

En fait, la position que présente ici Heidegger ne consiste pas tant dans la tentative de réunir deux positions métaphysiques en apparence irréconciliables (comme c'est le cas chez Marion) que dans une manière de thèse «transitoire » entre l'analyse des années vingt (infra, $\sqrt{ } 1$ ) et l'interprétation qu'il va déployer au début des années quarante (infra, \3).

1. C’est ainsi que l'on doit comprendre l'aspect mathématique de la connaissance: «Le mathématique, c'est cela que nous connaissons déjà proprement "aux" choses, que nous ne commençons pas par extraire des choses, mais que d'une certaine manière nous portons déjà en nous-mêmes » $(G A, 41,74 ;$ tr. 86).

2. Ibid., 104; tr. 114.

3. Ainsi que dans les SS 1927 (GA, 24) et WS 1929/30 (GA, 29/30).

4. $G A, 41,109$; tr. 119 .

5. Ibid., 110; tr. 120.

6. Ibid., 110-111; tr. 120. 
Nous verrons, en effet, que Heidegger tente, dans ses cours sur le nihilisme européen ${ }^{1}$, de présenter la pensée cartésienne en faisant fi de son aspect scolastique - donc, en ne tenant plus compte de la compréhension de l'être comme esse creatum.

La métaphysique, rappelons-le, interroge à chaque fois l'étant en totalité et l'être de l'étant. Selon le cours du semestre d'hiver 1935/36, l'étant se comprend encore chez Descartes et dans le cours des Temps Modernes comme ens creatum, faisant de Dieu la «source créatrice de tout étant $»^{2}$. La représentation chrétienne de l'étant est donc conservée mais son étude est désormais orientée sur le trait mathématique fondamental : la théologie devient theologia rationalis, la cosmologie, cosmologia rationalis, et la psychologie, psychologia rationalis ${ }^{3}$. Or, il ne faut pas croire que seule la forme de la métaphysique ait été modifiée. L'homme, en tant que source de la pensée, devient le nouveau point de référence de l'interrogation métaphysique. Bien que la metaphysica specialis - théologie, cosmologie, psychologie - n'ait été modifiée qu'au niveau de sa forme, la metaphysica generalis l'ontologie - qui s’interroge sur l'étant en général, a été remplacée : «il faut que soit décidé en elle principiellement (grundsätalich) selon des axiomes, et selon le premier axiome, selon le schéma du poser et du penser en général, ce qui appartient à l'étant comme tel en général, ce qui détermine et délimite la choséité d’une chose (die Dingheit eines Dinges) en général ».

Sans employer le concept d'onto-théologie ${ }^{5}$, Heidegger tente de concevoir la métaphysique cartésienne comme la rencontre de deux pensées difficilement conciliables. Il semble donc bel et bien que Heidegger ait envisagé une telle cohabitation. Mais cette interprétation va être bien vite abandonnée au profit d'une lecture uniquement axée sur l'aspect égologique ou mathématique de la pensée de Descartes. Les cours sur Nietzsche du début des années quarante vont, en effet, tenter de montrer que l'interprétation de la res cogitans que donne Descartes dans le langage de la scolastique (substantia finita, ens creatum) est « extérieure et insuffisante $»^{6}$.

1. $G A, 6.2$.

2. $G A, 41,109-110 ;$ tr. 119 .

3. Ibid., 110; tr. 119.

4. Ibid., 112; tr. 121.

5. Rappelons que les noms de metaphysica specialis et de metaphysica generalis ne coïncident pas avec ceux d'ontologie et de théologie dans le concept heideggérien de la métaphysique comprise comme onto-théologie. Voir à ce sujet, GA, 6.2, 313-314; tr. 279.

6. Ibid., 144; tr. 132. 


\section{\3. L'onto-théologie de la repraesentatio: ens in quantum cogitatum}

La fin des années trente et le début des années quarante ont été pour Heidegger l'occasion d'entrer dans une explication décisive avec Nietzsche. Cette mise en rapport de la pensée nietzschéenne avec celle des Temps Modernes sert de motif à une description in extenso de la métaphysique de Descartes. Ici encore, ce n'est pas le modèle onto-théologique que Heidegger emploie pour caractériser cette métaphysique, mais un schéma quadripartite qui avait déjà été exposé en $1938^{1}$. Abordant le «projet de l'être de l'étant» tel qu'il se présente chez Descartes, Heidegger déploie une lecture originale de cette métaphysique. Cette interprétation de la pensée cartésienne laisse de côté son aspect scolastique et soutient que l'être ne s'y comprend pas tant comme substantialité que comme représentéité (Vorgestelltheit). Si Heidegger a tenté au milieu des années trente de réconcilier les deux faces du cartésianisme (la représentation chrétienne de l'étant et le trait mathématique de la pensée), il ne va considérer, dès les années quarante, que l'aspect fondamentalement moderne de cette pensée : l'être comme représentéité du sujet qui représente.

Se détournant de l'idée selon laquelle Descartes n'aurait fait que reprendre l'ontologie de ses prédécesseurs, Heidegger lit désormais les Méditations en y cherchant une métaphysique nouvelle, fondée en la proposition essentielle cogito, ergo sum. Bien que celle-ci soit généralement traduite par «Je pense donc je suis », Heidegger préfère rendre le cogitare par «se représenter» (Vor-sich-stellen), c'est-à-dire la capacité unique d'avoir une représentation de quelque chose mais aussi de pouvoir se représenter soi-même. Si Heidegger insiste autant sur le rôle de la représentation de soi chez Descartes, c'est qu'il y voit le fondement de toutes les représentations - la première et originelle représentation a la forme d'un «Je me représente ». La définition même du cogitare, en tant qu'il se déploie originellement dans l'ego cogito, se donne comme l'acte de représenter quelque chose à celui qui se représente, l'ego. Ainsi, dans chacune de ses représentations, le moi est toujours déjà implicitement contenu ${ }^{2}$. C'est en ce sens que doit être comprise la formule heideggérienne cogito me cogitare: "Tout ego cogito est: cogito me cogitare; tout "je (me) représente quelque chose" du même coup "me" représente, moi qui me représente quelque chose (devant moi dans mon représenter) $»^{3}$.

1. Voir la conférence Die Zeit des Weltbildes publiée in Holzwege, GA, 5; tr. L'époque des «conceptions du monde » publié in Chemins qui ne mènent nulle part.

2. Cette importance du moi pour l'acte de (se) représenter quelque chose est reconnue par Descartes qui

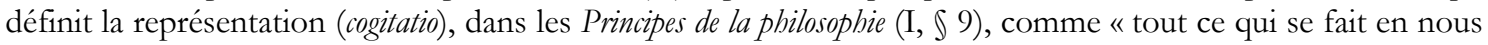
de telle sorte que nous l'apercevions immédiatement et par nous-mêmes » (AT, IX, II, 28).

3. $G A, 6.2,135 ;$ tr. 124 . 
La réponse de Descartes à l'interrogation métaphysique est donnée par la place qu'occupe la proposition cogito, ergo sum comme première et plus certaine de toutes les connaissances. C'est, soutient alors Heidegger, par la méditation de cette proposition et de son importance dans l'édifice métaphysique cartésien que nous parviendrons à comprendre authentiquement cette métaphysique. Il est désormais possible - contrairement à ce que défendait Heidegger dans les années vingt - de trouver dans la pensée cartésienne une véritable et originale réflexion sur l'être de l'étant et une tentative de déterminer ontologiquement ce que signifie «sum» dans « ego sum ».

La signification du sum de l'ego est explicitée dans la co-appartenance insigne de l'acte de représenter (ego cogito) et de l'existence d'un moi (ego sum) qui représente. Du moment que l'étant est appréhendé en tant que représenté (et non plus en tant que créé, in quantum ens creatum), le « je » comme représentateur, comme ce qui se tient à l'encontre de l'objet représenté, " est si certainement disposé à la représentation qu'aucune induction, si concluante fût-elle, ne saurait jamais atteindre la certitude de cette disposition devers soimême de celui qui (se) représente ${ }^{1}$. La compréhension de l'étant comme ens cogitatum est aussi peu compatible avec l'inexistence du « je » représentant que peut l'être, par exemple, celle de l'étant comme ens creatum avec l'inexistence du creator. En ce sens, il est erroné, soutient Heidegger, d'interpréter le cogito, ergo sum comme la déduction du je suis à partir du je pense. L'existence du je, son existo ou son sum, est en fait posé tout aussi originellement que sa pensée, son cogito. C'est pourquoi Heidegger préfère la formulation «cogito-sum » qui n'a plus l'apparence d'un syllogisme et ne fait qu'énoncer la co-appartenance, conjuguée à la première personne du singulier, de l'être (sum) et de la représentation (cogito) - soit, la thèse de Descartes sur l'être ${ }^{3}$. Cogito-sum : le représenter n'a de réalité qu'avec la position ou l'existence d'un je qui représente - la position ou l'existence du je n'a de réalité que dans l'acte réflexif du représenter qui se représente lui-même. À partir de la pleine essence de la représentation telle que définie ici se détermine enfin celle de l'être : il se donne comme représentéité.

Comment alors interpréter la formule sum res cogitans qui fit écrire à Heidegger, en 1927, qu'elle menait Descartes à penser l'ego sur le mode d'être des objets, comme res? En admettant que Descartes ait su repenser l'essence de la choséité comme représentéité, rien

1. Ibid., 142; tr. 130. Nous retrouvons cette thèse au $\int 49$ de la première partie des Principes de la philosophie dans lequel Descartes compte au nombre des premières vérités (aux côtés du principe de contradiction) «que celui qui pense [is qui cogitat $=$ celui qui se représente] ne peut manquer d'être ou d'exister pendant qu'il pense » (AT, IX, II, 46).

2. $G A, 6.2,142$; tr. 130 .

3. Si Heidegger modifie la formulation de la thèse cartésienne, il se défend bien d'en modifier le contenu. Selon lui, « le grand obstacle à la juste compréhension de la proposition [cogito, ergo sum] c'est la façon dont Descartes l'a formulée » (Ibid., 140; tr. 128). 
ne nous empêche alors d'admettre que l'ego soit bel et bien une res cogitans, c'est-à-dire un ens cogitatum cogitans, un étant représenté représentant ${ }^{1}$. Le mode d'être des étants en général et de l'ego en particulier ayant été repensé radicalement, il n'est plus possible de soutenir que l'ego souffre d'une indétermination ontologique. Heidegger revient, du reste, sur cette idée, treize ans après Sein und Zeit, mais pour rectifier le tir: «Traduite mot à mot [la formule sum res cogitans] signifie: je suis une chose pensante. Ainsi, l'homme se verrait défini tel un objet donné (ein vorhandener Gegenstand), à ceci près que la propriété du "penser" lui serait attribuée en tant que caractère distinctif. Toutefois cette manière d'interpréter la proposition [soit celle que Heidegger défendait dans Sein und Zeit oublierait que le sum se détermine en tant que ego cogito. Elle oublierait que la res cogitans, conformément au concept de la cogitatio, signifie dans le même temps : res cogitata: soit: ce qui se re-présente soi-même (das sich selbst Vorstellende). Elle oublierait que ce re-présenter-soi-même contribue à constituer l'être de cette res cogitans $»^{2}$.

La critique qui était au centre de la «Discussion herméneutique de l'ontologie cartésienne» de Sein und Zeit se fonde donc sur une lecture erronée de la pensée cartésienne ${ }^{3}$. Cette critique oubliait que Descartes a fourni à l'histoire de la métaphysique une réponse originale à la question de l'étant et qu'il n'a pas employé le syntagme ego sum de façon indécise mais en un sens particulier qui a modifié la compréhension de l'être des philosophies ultérieures.

Heidegger soutient désormais qu'avec Descartes, les fondements de la métaphysique ont été radicalement repensés. Après avoir sévèrement critiqué sa reprise non questionnée de la terminologie scolastique, Heidegger reconnaît enfin la profondeur de sa pensée. Mais on ne peut nier que Descartes ait conservé certains aspects de la pensée qui le précède. Ne serait-ce que dans l'emploi - hésitant, il est vrai - du lourd terme de «substance ${ }^{4}$, la présence de l'ontologie scolastique se fait encore sentir. Heidegger propose toutefois une solution à cette difficulté : «Descartes suggère lui-même une

1. L'ego est d'ailleurs le seul étant qui puisse réclamer un tel titre - d'où sa singularité. Un parallèle avec le Dasein s'impose : il est le seul étant qui soit doté d'une compréhension de l'être. L'ego, quant à lui, est la seule représentation - il l'est dans la mesure où c'est comme «représentation de soi » qu'il se connait - qui puisse avoir des représentations.

2. $G A, 6.2,144 ;$ tr. 132 .

3. Marion, dans Sur le prisme métaphysique de Descartes, soutient qu'il faut « récuser la thèse soutenue par Heidegger dans Sein und Zeit» puisque l'essence de la substance se dit à partir de l'ego qui, "loin de subir l'indétermination de la substance, lui impose ses propres déterminations [...] La substance a d'abord l'essence d'un je, parce qu'elle se déduit d'un ego » (177). Heidegger avait en fait déjà « récusé » sa thèse dès 1940.

4. Ce n'est qu'à partir des Méditations et, plus tard, dans les Principes de la philosophie, que Descartes emploie ce concept. Il est à noter que dans les Regulae ad directionem ingenii, les genres et les catégories - et donc la substance - avaient été proscrits. Voir, pour l'histoire de l'apparition du concept de substance chez Descartes, les Questions cartésiennes II - Sur l'ego et sur Dieu (PUF, Paris, 1996, 89 sq.) de Jean-Luc Marion. 
interprétation extérieure et insuffisante de la res cogitans, pour autant qu'il s'exprime doctrinalement dans le langage de la scolastique médiévale et répartit l'étant dans sa totalité en substantia finita et substantia inifinita. (...) Le domaine de la substantia finita est l'ens creatum. Celui-ci, Descartes le divise en res cogitantes et res extensae. De la sorte tout étant est considéré à partir du creator et du creatum et la nouvelle détermination de l'homme par le cogito sum ne se voit pour ainsi dire qu'inscrite dans le vieux cadre scolastique. Nous avons ici l'exemple le plus tangible de la superposition de la pensée antérieure sur un nouveau début de la pensée métaphysique $»^{1}$. La res cogitans comprise comme ens creatum élimine ce que le cogito sum venait à peine de conquérir. La nouvelle détermination de l'homme (comme res cogitata cogitans) se trouve anéantie par sa ré-inscription dans le cadre scolastique. Cette interprétation extérieure et insuffisante qui est à l'origine de la critique heideggérienne des années vingt devrait donc être rejetée comme non essentiellement cartésienne. Seule la détermination de l'étant in quantum ens cogitatum permet de saisir l'originalité de la pensée de Descartes.

Le dernier mot de Heidegger sur la constitution de la métaphysique cartésienne semble donc se rapprocher de ce que Marion appelle la première figure de l'onto-théologie, celle de la cogitatio. Soutenant, comme nous venons de le montrer, que l'être se dit, à partir de Descartes, selon la représentéité, Heidegger voit désormais dans l'ego le fondement d'une onto-théologie nouvelle, à l'origine de la métaphysique de la subjectivité. L'étant (l'ôv) se comprend désormais comme ens cogitatum - ce qui fait de l'ego (fondement du cogitare) l'étant suprême (le $\theta \varepsilon \hat{\imath} \hat{o v})$, l'unique ens cogitatum cogitans ${ }^{2}$.

Or, ces différentes interprétations de la métaphysique cartésienne correspondent à différentes époques dans la pensée heideggérienne et, à certains égards, servent différentes fins. Les raisons qui ont poussé Heidegger à se confronter à la métaphysique cartésienne dans Sein und Zeit ne sont pas les mêmes que dans les années trente et quarante. Chacune de

1. $G A, 6.2,144$; tr. 132. Heidegger considère donc que la notion cartésienne de substance est une manière de «survivance» d'un mode de pensée révolu et non pas un concept original redéfini à la lumière d'une nouvelle compréhension de l'être. Â aucun moment il ne va tenter - comme le fait Jean-Luc Marion dans Sur le prisme métaphysique de Descartes (\$ 13) - de montrer que Descartes «tire» en fait le concept de substance de l'idée qu'il a de lui-même (Descartes écrit, en effet, dans la Méditation $I I I^{e}$, AT, IX, 35 : «Quant aux idées claires et distinctes que j'ai des choses corporelles, il y en a quelques-unes qu'il semble que j’ai pu tirer de l'idée que j'ai de moi-même, comme celle que j'ai de la substance, ...»).

2. Nous pourrions aussi utiliser l'expression cogitatio sui de Marion qui fait écho à la causa sui de l'ontothéologie de la causa. 
ces interprétations est motivée par des fins bien précises qui, en un certain sens, nous permettent de justifier l'évolution qui vient d'être exposée.

\section{Conclusion}

Descartes et la question de l'être

Malgré cette nouvelle lecture de Heidegger qui soutient que la pensée cartésienne constitue bel et bien un renouveau de la métaphysique, il ne faut pas pour autant croire que la question de l'être ait reçu par là une nouvelle formulation. Même si Descartes comprend l'être de l'étant d'une nouvelle façon, il ne thématise jamais lui-même dans ses textes sa propre compréhension de l'être. L'être ne se dit peut-être plus selon la substantialité mais le regard irrémédiablement théorique que pose Descartes sur les choses ne lui permet pas de penser l'étant - et tout d'abord, l'ego - autrement que selon l'idée de l'être comme ständige Vorbandenheit. Le mode d'être de la représentéité tout comme celui de la substantialité ne sont en fait que des modes d'être attachés à ce qui subsiste de l'étant à travers le temps. En développant une nouvelle ontologie sans pour autant soulever la question de l'être, Descartes apparait en fin de compte dans l'histoire de la métaphysique comme un barrage supplémentaire au déploiement de cette question ${ }^{1}$. Mais comme nous l'avons exposé dans cet article, les motifs de cette critique de Descartes ne sont pas restés les mêmes des années vingt aux années quarante.

Dans les années vingt, l'essentiel de l'examen de la pensée cartésienne portait sur le fait que Descartes n'avait pas su renouveler l'interrogation métaphysique bien qu'il en ait modifié l'orientation de façon fondamentale (elle ne traite désormais plus de la nature mais de la pensée). Récupérant une ontologie à la mesure de l'ancienne orientation (sur la nature), l'étant dans son ensemble - mais tout d'abord l'ego - se trouve indéterminé quant à son mode d'être. L'importance que revêt alors cette confrontation tient au projet même de Sein und Zeit - celui de l'ontologie fondamentale. Tentant de poser à neuf la question du sens de l'être en s'appuyant sur le lieu même de la question (le Dasein), Heidegger doit assurément différencier cette entreprise de celle de Descartes qui, malgré tout, partage avec elle une certaine ressemblance. Descartes souhaite, en effet, refonder le savoir dans son ensemble en partant du sujet connaissant comme témoin privilégié du déploiement de la

1. Cela, Heidegger l'a soutenu depuis l'époque de Sein und Zeit jusqu'aux tout derniers séminaires. Voir, par exemple, «Le séminaire de Zähringen » de 1973, publié in Seminare (1951-1973), GA, 15; tr. Questions III et IV, 470. 
connaissance. Heidegger, quant à lui, souhaite « refonder la métaphysique ${ }^{1}$ en partant du Dasein comme témoin de la question de l'être. Bien que ce parallèle soit très approximatif, l'entreprise de Descartes représente depuis toujours pour Heidegger le piège dans lequel ses prédécesseurs sont tombés ${ }^{2}$. C'est pourquoi l'indétermination ontologique de l'ego devient un thème d'une importance majeure dans les cours des années vingt, puis dans Sein und Zeit. Le Dasein sera « ontologiquement déterminé » ou ne sera pas!

Mais ce projet ambitieux restera inachevé. Dès les années trente, Heidegger a de nouveaux projets et la «métaphysique du Dasein» sera laissée de côté. Tâchant de retracer l'histoire de la métaphysique - plutôt que de s'y inscrire -, Heidegger va encore une fois se mesurer à la pensée cartésienne. Interprétant la pensée de Nietzsche à la lumière de la nouveauté de la métaphysique moderne, Heidegger propose une lecture de la pensée cartésienne qui est alors fort éloignée de celle qu'il avait déployée dans les années vingt. Descartes fait dès lors figure de novateur dans le cours de l'histoire de la métaphysique en ayant transformé la compréhension de l'étant de la substantialité à la représentéité. Autrement dit, la métaphysique de la subjectivité qui a mené à l'achèvement hégélien de la métaphysique (dans l'absolu) mais aussi à son dépassement nietzschéen (dans la volonté de puissance) prend sa source dans la compréhension cartésienne de l'être de l'étant.

Certes, une différence dans les fins ne saurait suffire à justifier une différence dans l'interprétation. Pour les interprètes de la pensée cartésienne, il va de soi que les bouleversements qu'a pu connaitre l'œuvre de Heidegger n'ont pas d'importance. Mais pour ceux qui lisent Heidegger et tentent de comprendre la rigueur interne de sa pensée, les différences d'interprétations qui existent entre les textes des années vingt et ceux de la fin des années trente sont fondamentales.

1. C'est le jugement que lui-même portera en 1929 sur l'entreprise de Sein und Zeit: «La question de l'être, question fondamentale de l'instauration du fondement de la métaphysique, est le problème de Sein und Zeit» $(G A, 3,203$; tr. 257).

2. Comme on le sait, Husserl lui-même n'a pas su sortir de la sphère de la conscience. Heidegger, dès le début des années vingt (voir Phänomenologische Interpretationen zu Aristoteles. Einführung in die phänomenologische Forschung, $G A, 61,173)$ avait déjà identifié la pensée cartésienne comme l'obstacle le plus important au déploiement d'une philosophie authentique. 